\title{
ACTITUDES DE ESTUDIANTES Y PROFESORADO SOBRE LAS CARACTERÍSTICAS DE LOS CIENTÍFICOS
}

\author{
MANASSERO MAS, MARÍA ANTONIA ${ }^{1}$ y VÁZQUEZ ALONSO, ÁNGEL \\ ${ }^{1}$ Departamento de Psicología. Universidad de las Islas Baleares \\ E-mail: dpsamm0@ps.uib.es \\ ${ }^{2}$ Departamento de Física, Universidad de las Islas Baleares \\ E-mail: dfsava0@ps.uib.es
}

\begin{abstract}
SUMMARY
This study assesses some students' and teachers' views toward scientists' values about the scientists' motivation to investigate, impartiality and objectivity, honesty, social activities and relationships, and patience and self-determination abilities. On the whole, teachers' and students' attitudes are too similar, except in the case of scientists' impartiality and objectivity and sometimes ambivalence. Claims to explicitly include the history, epistemology and sociology of science in school curriculum and teachers' training are suggested, as a means to improve the teaching and learning of science.
\end{abstract}

\section{INTRODUCCIÓN}

Este estudio aborda aspectos concretos de la sociología interna de la ciencia, centrados en algunas características de los científicos que son consecuencia de los valores y la organización de la comunidad científica. Se realiza una aproximación sencilla al conocimiento de las actitudes básicas de estudiantes y profesorado sobre los valores característicos del trabajo de los científicos, en el marco de la complejidad de la gran ciencia actual.

La epistemología positivista de la ciencia considera el conocimiento científico neutro, libre de valores, ni bueno ni malo y, por supuesto, no influido por ideologías, intereses o razones coyunturales de las personas de ciencia. Desde esta epistemología, el estereotipo del científico quedaba como una persona de mente abierta, imparcial y objetiva en sus apreciaciones, honrada, motivado por el afán de conocer y con un tesón infinito en la dedicación a su labor. Sin embargo, ante la evidencia histórica de sesgos, errores y fraudes en la ciencia, el neopositivismo ha hilado más fino, distinguiendo el contexto de descubrimiento, donde se admite que podrían actuar y tener cabida sesgos de la naturaleza humana, valores, intereses, etc. y el contexto de justificación, donde la validación del conocimiento mismo queda a salvo de la influencia de las anteriores debilidades humanas (Reichenbach, 1966).

En parte como consecuencia de esta filosofía, las disciplinas científicas se ofrecen a los estudiantes en facultades, institutos y escuelas de una manera parcial, excesivamente centradas en los contenidos y conceptos de cada disciplina (leyes, teorías, ecuaciones y formalismos), los cuales llenan los libros de texto y los manuales científicos y la mayor parte del tiempo empleado en las clases, como coartada de la objetividad y la neutralidad que el positivismo lógico predica del conocimiento científico y olvidando los factores contextuales de cualquier actividad humana. Este enfoque de la ciencia de los 
libros de texto y las clases, centrado en los contenidos como conocimientos elaborados (Gil, 1993), borra el proceso histórico a través del cual han sido construidos esos conceptos, produce una imagen de la ciencia en la que se omiten los problemas (a veces, sociales) que originaron el conocimiento, la evolución y las controversias, las limitaciones o las perspectivas abiertas y, por ello, deforman el papel del científico como protagonista y responsable principal de la ciencia.

La imagen ahistórica de la ciencia también cercena la influencia de la sociedad sobre la ciencia y los científicos, y también la influencia de la ciencia sobre la sociedad, así como las relaciones con la tecnología (CTS). Ciertamente, muchos de los rasgos de esta influencia son hoy tan obvios (progreso en la salud pública, la vivienda, la energía, los alimentos, los transportes, etc.), y están tan presentes en la vida diaria de tantas personas en el mundo que parece una petulancia hablar de ello. Por otro lado, la cara negativa del progreso científico como capacidad de autodestrucción, deterioro del medio ambiente, errores, fraudes, etc. forma parte importante de la conciencia actual, pero que tampoco es reconocida suficientemente en las instituciones científicas.

La pérdida sustancial del elemento histórico produce una ciencia escolar y académica que no se corresponde con la ciencia real, con sus contingencias diarias, sus presiones, sus casualidades, sus servidumbres, sus limitaciones humanas, etc., trasmitiéndose así una imagen inexacta y distorsionada de la ciencia y los científicos. Estos conceptos ahistóricos de la ciencia, ignorantes de la influencia social en la génesis del conocimiento científico, producen en los estudiantes concepciones y actitudes ingenuas (Vázquez y Manassero, 1997) que se manifiestan en imágenes estereotipadas de la ciencia y de los científicos y que se utilizan ingenua y profusamente en la literatura, el cine o los medios de comunicación. El estereotipo del «científico chiflado», malvado, egoísta, absorbido por sus investigaciones, aislado de la sociedad en su torre de marfil, excéntrico, insensible, frío y cerebral, obsesionado por su trabajo y con alcanzar sus objetivos a cualquier precio, malhumorado $\mathrm{y}$, a veces, insano ha sido repetidamente identificado (Kahle, 1989; Mason, Kahle y Gardner, 1991; Matthew, 1991; McAdam, 1990; Newton y Newton, 1992; Schibeci y Sorenson, 1983; Weart, 1988), aunque otros estudios comienzan a delinear una imagen menos estereotipada y más realista (Boylan, Hill, Wallace y Wheeler, 1992; Matthews, 1996; Schibeci, 1986; Vázquez y Manassero, 1998a).

Puesto que el pensamiento positivista ha tenido una influencia penetrante, especialmente en los ambientes científicos y técnicos, en los que se han formado la mayoría de los profesores y éstos, a su vez, pueden influir en las actitudes y opiniones de los alumnos que educan, diagnosticar las actitudes generales sobre las características de los científicos constituye una medida del reconocimiento de la influencia de factores sociales y personales en la ciencia y la tecnología y, a la vez, también un test del arraigo del pensamiento positivista.

\section{La sociología de la ciencia, un marco conceptual alternativo al positivismo}

Cualquier aproximación histórica a la ciencia muestra que ésta es un fenómeno social. Los análisis sociológicos de la ciencia han estudiado la influencia de los factores sociales y personales sobre aquélla (influencias mutuas ciencia-sociedad), así como el sistema de valores de la comunidad científica, condicionado por las características del sistema de recompensas y reforzadores y por la estratificación interna (sociología interna de la comunidad científica). El programa fuerte de la sociología del conocimiento defiende la tesis de la determinación del conocimiento científico por factores sociales y personales.

Para entender el desarrollo de la sociología de la ciencia debe tenerse presente el gran cambio producido en la ciencia en la segunda mitad del siglo xx caracterizado por la explosión informativa, de recursos humanos $\mathrm{y}$ financieros que caracterizan la «gran ciencia» actual, en contraste con la «pequeña ciencia», nombre con el que se denomina a la ciencia existente antes de la segunda guerra mundial. Sociológicamente, además de una explosión cuantitativa exponencial, la «gran ciencia» trae cambios cualitativos importantes en algunos rasgos relevantes de la ciencia (Sánchez Ron, 1992). Los análisis sociométricos y bibliométricos de la literatura, la población y los presupuestos de la ciencia muestran que el crecimiento de las publicaciones científicas, como modo básico de reconocimiento y recompensa dentro de la comunidad científica respecto a sus miembros («publica o perece»), junto con el incremento de los trabajos en colaboración de múltiples autores, pasando de la autoría individual a la autoría múltiple, constituyen una de las transiciones más violentas de los últimos años en la evolución científica (De Solla-Price, 1973, pp. 140-142). Este rasgo es, en parte, consecuencia de una reconversión e integración de los científicos aislados en grandes equipos de trabajo y por las más estrechas relaciones entre los diferentes equipos que investigan en una misma área de conocimiento y que dan pie a la constitución de los denominados colegios invisibles (Knorr-Cetina, 1981).

La integración de los científicos en grandes equipos de trabajo especializados e interdisciplinares no es ajena a la gran magnitud de los proyectos tecnocientíficos (carrera espacial, grandes aceleradores de partículas, proyecto del genoma humano, investigación militar, etc.), que requieren la colaboración concertada de gran cantidad de técnicos y científicos, necesitan una programación cuidadosa y una financiación exorbitante para realizar experimentos de mucho tiempo. La realización de estos proyectos es imposible sin recurrir a la única fuente capaz de proveer la financiación necesaria, como los gobiernos y las agencias oficiales, y cuyo resultado es el progresivo control social de la ciencia y la tecnología por los gobiernos y los organismos públicos. Al mismo tiempo, las condiciones de experimentación están en el límite de sensibilidad de los instrumentos de trabajo, de modo que la relación entre evidencia empírica y conceptos es más débil que nunca y la creatividad y la imagina- 
ción se hacen instrumentos cotidianos en el trabajo de los científicos (Campanario, 1999).

En este contexto de gran ciencia se desarrollan los valores y rasgos de organización para garantizar su pervivencia como institución social, además de la eficacia demostrada para resolver problemas específicos, y que a nivel individual determina la conducta profesional, las motivaciones y los valores de los científicos como consecuencia del refuerzo obtenido de los intercambios científicos.

El primer factor, los valores, son el conjunto de reglas, prescripciones y presupuestos, profesados por los científicos, cuya adhesión obedece a las exigencias metodológicas de la actividad científica y que constituye el elemento de socialización y sanción para los miembros de la institución. Los elementos básicos de los valores científicos (Merton, 1977) son el comunismo (los descubrimientos son propiedad de toda la comunidad), el universalismo (todos los científicos son iguales, con las mismas prerrogativas y servidumbres), el desinterés (el carácter público y la rendición de cuentas ante la comunidad exige la renuncia a otro beneficio que no sea el trabajo bien hecho) y el escepticismo organizado (el examen independiente y ecuánime de los resultados), resumidos en el acrónimo CUDEOS. El autor añadió más tarde otros dos nuevos valores: humildad (consecuencia del desinterés y el escepticismo) y originalidad (consecuencia del comunismo y el sistema autónomo de recompensas).

El intercambio de información es el segundo factor de la institucionalización de la ciencia identificado por Merton, a la vez motor de la actividad científica y medio para la búsqueda del reconocimiento a través del sistema de recompensas y reforzadores mediante los cuales se ejerce el control de la institución. El reconocimiento es proporcional a la aportación personal a la comunidad y se vehicula a través de recompensas honoríficas, como la prioridad en la autoría, la eponimia (atribución del nombre del descubridor a su descubrimiento), el ingreso en las academias de la ciencia, la otorgación de premios y distinciones (el Nobel sería el culmen de una carrera), la elección para cargos simbólicos y materiales y, en general, cualquier tipo de reconocimiento de los colegas. De esta manera, el sistema de intercambio en la comunidad científica actúa como un reforzador de las normas y valores de la comunidad a través de las recompensas instituidas.

Otro factor de la institucionalización de la ciencia se refiere a las pautas de estratificación en la organización (Merton, 1977). La distribución de las recompensas causa desigualdades entre los científicos, que se concretan en tres hechos denominados el «efecto del sillón 41 » (la academia de ciencias sólo tiene 40 sillones), el «efecto de trinquete» (una vez alcanzado un nivel, nunca se baja de él aunque llegue una persona mejor dotada) y el «efecto Mateo» (acumulación de recompensas en unos pocos científicos y su negación a otros muchos), lo cual es la fuente primigenia del tipo de organización social interna del sistema científico. El efecto Mateo está omnipresente en la ciencia y es una consecuencia del propio sistema de evaluación del trabajo científico, que refuerza positivamente la acumulación de recompensas, y negativamente su carencia, de modo que, aunque el rango y la autoridad se adquieren legítimamente mediante realizaciones pasadas exitosas, una vez adquiridas pueden amplificar inercialmente la reputación profesional de los autores, a la par que ensombrece y reduce la de otros, atrayendo recursos y dotaciones, retrayéndolos a otros, y originando efectos perniciosos, como los juicios sesgados de estas personas y una cierta inmunidad a las críticas. Esto lleva a la constitución de gerontocracias científicas que controlan una gran parte de la organización, como evaluadores de programas y proyectos, asignadores de recursos, censores de revistas, organizadores de congresos, etc.

El análisis mertoniano se considera hoy una primera aproximación clásica superada por la realidad. El debate crítico sobre los CUDEOS se ha centrado en el idealismo y la generalidad de la propuesta, que podría estar alejado de la realidad ética de la organización científica. Las contradicciones existentes entre algunos de los valores (p.e., el ansia de recompensas es contrario al desinterés o al escepticismo) pueden llevar a conductas desviadas de los CUDEOS, como el fraude o el plagio. Uno de los fraudes más citados es la falsificación de pruebas, que puede consistir simplemente en la selección sesgada de los datos, aceptando los que confirman la propia hipótesis y desechando los contrarios a ella; la calumnia de plagio o acusación falsa de plagio hacia otro y el plagio inconsciente son otras formas frecuentes de conductas desviadas.

La comunidad científica es un grupo altamente organizado y jerarquizado, coexistiendo, en su interior, una gran diversidad de intereses, posiciones, expectativas, etc. de los distintos miembros y niveles, que enmascara, a menudo, divergencias y tensiones. Esta diversidad surge de la división del trabajo que se practica en su seno, que conduce a una estratificación social en «clases» (p.e., el trabajo de un estudiante es diferente del trabajo del jefe de equipo) y a una clara distinción entre los proyectos y su aplicación práctica, y que es un reflejo de la división entre la ejecución del trabajo y los objetivos últimos de éste, característicos de la sociedad industrial. Se trata de producir resultados científicos sin preocuparse por su finalidad, ni plantearse sus implicaciones sociales, lo cual crea una mentalidad de estricta separación entre el trabajo profesional científico y el resto de la vida, que produce un modelo social de la doble cultura, ya criticada por Snow (1987). Esta filosofía tiene implicaciones directas en la formación de los científicos, a quienes se presenta una ciencia donde se han borrado los orígenes históricos y sociales de los conocimientos científicos, dejando éstos reducidos a su estricto contenido técnico, descontextualizados. Esta formación pretende hacer de la ciencia un conocimiento neutral, por aislado, y evitar los posicionamientos críticos respecto a ella.

Sin embargo, la comunidad científica tiene escaso poder decisorio real y directo en el concierto social. Como 
tiene una necesidad muy clara, que es la financiación de la investigación, busca aliados que detenten ese poder y que estén interesados en sus productos. La industria y los militares se convierten en los poderosos aliados que subvencionan la actividad científica, a través de la industrialización y la militarización de la ciencia, que son muy cuestionadas, especialmente la última. Estas alianzas determinan que la comunidad científica, como grupo, no es neutra y desinteresada, sino que está mediatizada por estos poderosos factores. Siguiendo la inercia de los sistemas sociales, la comunidad científica tiende a cristalizar los intereses internos creados por el propio sistema, que a la vez estructuran su propio espacio de poder y de clientela y se convierten en normas burocráticas de funcionamiento interno. Como en todos los grupos, los vínculos entre sus miembros generan fidelidad a los valores del grupo y corporativismo, en defensa de los intereses del grupo, con la consiguiente ocultación de estos intereses y la inmediata defección de los infieles en aras de la cohesión del grupo (Campanario, 1999).

En suma, la realidad constata que la vida científica no se organiza, de hecho, como una comunidad de iguales y de intercambio de información sin limitaciones. Existen elites que evalúan y juzgan la distribución de cargos y recursos; el secretismo afecta a la globalidad de la actividad, para proteger el reconocimiento de propiedad y autoría, y en algunos casos es permanente (industria o militares); en vez de desinterés, existe una intensa competencia por publicar, por la prioridad de autoría, por conseguir medios y cargos, por la fama, etc.; los compromisos afectivos hacen que el escepticismo organizado se ejerza muy sesgada y emocionalmente: se es estricto hacia las tesis de los oponentes y benévolo para las propias. Si de la conducta global se pasa a las conductas individuales, Mitroff (1974) ha demostrado que la realidad de la conducta de los científicos se caracteriza por la ambivalencia normativa (se practican, a la vez, los valores CUDEOS y otros contravalores); cada valor CUDEOS tiene un contravalor, causa de las transgresiones y ambivalencia de la conducta de los científicos. Así, en oposición al universalismo, aparece el particularismo; contra el comunismo, aparece el secretismo; contra el desinterés, aparece la búsqueda del reconocimiento y el prestigio, justificada por el refuerzo del grupo de pertenencia; y contra el escepticismo organizado, aparece el dogmatismo organizado. Estos contravalores cumplen otras funciones necesarias para la institución y el progreso científico, como, por ejemplo, el mantenimiento de la cohesión de los grupos, que no siempre deben terminar en conductas desviadas y poco honrosas.

En apoyo de las tesis mertonianas, algunos estudios sobre la evaluación de manuscritos en revistas científicas por los censores, evaluación de calidad y excelencia en trabajos científicos o el reconocimiento de contribuciones al cuerpo científico demuestran que, a pesar de las desigualdades y la presencia de las gerontocracias en la organización, el sistema de estratificación de la ciencia no es excesivamente particularista y, en algunos casos, tiene un alto nivel de universalismo en las decisiones, ya que, por ejemplo, no se detectan esquemas preferencia- les en las evaluaciones que los censores hacen de los manuscritos (Lamo, González y Torres, 1994, pp. 476480). Todo ello demuestra que el problema de la estratificación social de la ciencia es algo más sofisticado y complejo, y difícilmente reducible a categorías simples.

Este estudio pretende conocer las actitudes de los estudiantes sobre algunas características básicas y concretas de los científicos poco analizadas en la literatura didáctica desde la perspectiva de la aplicación de un instrumento nuevo y validado empíricamente.

\section{MÉTODO}

\section{Muestra}

La selección de la muestra representativa de estudiantes se hizo por cuotas (grupos-clase), al azar, entre todos los niveles educativos, y proporcional a la población de cada nivel con un muestreo estratificado con afijación aproximadamente proporcional. La muestra de alumnado total está compuesta por 4.132 estudiantes (error muestral $\pm 4 \%$ ): a) universitarios (11\% de escuelas universitarias, $23 \%$ de licenciaturas, $8 \%$ titulados $[27 \%$ letras y artes, $43 \%$ sociales -psicología, derecho, economía-, $30 \%$ ciencias e ingenierías]; y b) secundarios, $22 \%$ ESO, $36 \%$ bachillerato y FP $[47 \%$ ciencias, $53 \%$ letras]). Las edades del $95 \%$ de la muestra están entre 14 años (edad más baja) y 27 años, con una mayoría de mujeres $(59 \%)$. La muestra que contesta cada uno de los seis cuestionarios aplicados originalmente, balanceada por género, se sitúa aproximadamente en torno a las 700 personas. En la tabla V se puede observar la distribución de la muestra del alumnado por grupos de exposición a la ciencia y género, muy semejante para los seis cuestionarios. La tradicional menor matrícula de alumnos en carreras de ciencias hace que el grupo de alta exposición a la ciencia sea el menos poblado.

La muestra de profesorado seleccionada fue contactada personalmente, cerca de tres veces superior a la muestra final, determinada por la decisión voluntaria y libre de remitir el cuestionario cumplimentado. La muestra final del profesorado es de 654 profesores (error muestral $\pm 4 \%$ ), que respondieron dos cuestionarios (318 y 336 en cada cuestionario) procedentes de primaria $(46 \%)$, secundaria (44\%) y universidad (10\%), de ciencias y letras (Tabla VI).

\section{Instrumento}

Se han aplicado un conjunto de cuestiones de papel y lápiz referidas a las características de los científicos del VOSTS: la motivación para trabajar (60111), imparcialidad y objetividad (60211), honradez (60226), las actividades y relaciones sociales de los científicos (60411) y las capacidades de paciencia y determinación (60421). Estas cuestiones han sido adaptadas de Aikenhead, Ryan y Fleming (1989) formando un banco de cuestiones aplicadas a un estudio más amplio con el objetivo de 
diagnosticar actitudes sobre todas las dimensiones CTS (Vázquez y Manassero, 1997).

Todas las cuestiones tienen el mismo formato de elección múltiple, que se inicia con un pie de unas pocas líneas, donde se plantea un problema, seguido de una lista de alternativas, cada una de ellas identificada correlativamente con una letra, que ofrecen todo un abanico plural de diferentes posiciones epistemológicas sobre el tema planteado, finalizando siempre con tres opciones comunes que recogen diversas razones para no elegir: 1) No entiendo la cuestión. 2) No sé lo suficiente sobre el tema para seleccionar una opción. 3) Ninguna de las opciones satisface básicamente mi opinión.

Las cuestiones aplicadas constituyen una nueva forma de prueba de opción múltiple «empíricamente desarrollada», construida sobre las respuestas previas de los propios estudiantes, y no las ideas del investigador, como suele ser habitual en otros instrumentos (Aikenhead, 1988). Con ello se evita la doctrina de la percepción inmaculada, es decir, la hipótesis implícita, en todos los estudios basados en cuestionarios de otros tipos, de que los encuestados y el investigador entienden $\mathrm{y}$ perciben el significado y el texto de las cuestiones exactamente de la misma manera, lo cual evita la ambivalencia y distorsión de los significados en las respuestas, y dota a las cuestiones de una validez inherente, no limitada a ningún modelo epistemológico particular sino ofreciendo todo un abanico plural de respuestas en cada cuestión (Aikenhead y Ryan, 1992).

\section{Procedimiento}

En cada cuestión, los encuestados deben seleccionar una sola opción, la que más se ajusta a la actitud del que responde. Cada estudiante contestó sólo uno de los seis cuestionarios en que se dividió el banco de cuestiones para facilitar la aplicación a los estudiantes (dos cuestionarios al profesorado), de modo que las cuestiones estudiadas han sido respondidas por muestras diferentes, aunque equivalentes entre sí por el sistema de distribución balanceada aleatoriamente de los seis cuestionarios en cada grupo-clase entre hombres y mujeres. La muestra de profesorado contestó dos cuestionarios. La aplicación de los dos cuestionarios del profesorado, los cuales contenían cuatro de las cuestiones correspondientes al tema objeto de este estudio $(60111,60211,60411$ y 60421), se realizó mediante autoadministración, de modo que la muestra final válida es aleatoria, pues corresponde a aquellos profesores que libremente decidieron participar en la encuesta.

La literatura didáctica informa que las actitudes relacionadas con los temas científicos dependen de diversas variables, entre las cuales la cantidad de educación científica recibida y el género son algunas de las más usuales (Vázquez y Manassero, 1995, 1996). La cantidad de educación científica recibida es importante y se ha operacionalizado en una variable denominada grado de exposición a la ciencia, mediante un sistema de puntuación proporcional a la cantidad de materias cien- tíficas cursadas por cada persona a lo largo de su currículo académico. Con esta variable, los licenciados y estudiantes del último curso de ingenierías, físicas o químicas alcanzan las puntuaciones más altas, mientras que los estudiantes de secundaria obligatoria, que todavía no han realizado la opción entre materias científicas y no científicas tienen la puntuación mínima. En base a estas puntuaciones la muestra total se ha dividido en tres categorías de exposición a la ciencia: baja, media y alta. Además, para el profesorado, se han considerado variables de licenciatura, edad y nivel educativo en que ejercen como docentes. Los análisis de las diferencias estadísticamente significativas entre grupos (chicuadrado, $\mathrm{p}<, 01)$ se han realizado en todos los casos controlando la variable exposición a la ciencia, para que las diferencias entre dos grupos se refieran siempre al mismo grado de exposición a la ciencia (Tablas V y VI).

\section{Resultados}

Se expone la distribución de las respuestas de alumnado y profesorado a cada una de las cuestiones acerca de las características de los científicos y las diferencias significativas entre algunos grupos de variables independientes. Una aproximación a las diferencias en las respuestas entre los diversos grupos generados por las variables independientes (exposición a la ciencia, género, nivel educativo, etc.) debe ser necesariamente sucinta, ya que su desarrollo detallado requeriría, como es obvio, un mayor espacio.

La exposición a la ciencia se ha considerado una variable de control en los análisis de las diferencias estadísticamente significativas entre grupos. Las cuestiones referidas a motivación para trabajar, imparcialidad y objetividad, honradez, y a actividades y relaciones sociales de los científicos muestran diferencias significativas según el grado de exposición a la ciencia del alumnado, pero no en el profesorado. En cambio, cabe resaltar que las diferencias en las respuestas a las cuestiones sobre la vida social y la determinación de los científicos no muestran diferencias según el grado de exposición a la ciencia.

\section{Motivación de los científicos según el alumnado (60111)}

La motivación de los científicos para hacer ciencia es juzgada por el alumnado (más de un tercio) desde una actitud un poco imprecisa, como un asunto muy personal, que varía de un científico a otro y que, por ello, no es posible generalizar. Sin embargo, una cantidad significativa (menos de un tercio) opina que la principal motivación de los científicos es descubrir e inventar para conocer y en beneficio de la sociedad. Esta última razón vuelve a repetirse en otra alternativa con una frecuencia relevante, de modo que se podría considerar, acumulando las frecuencias de ambas opciones, que la creencia mayoritaria del alumnado es que los científicos trabajan motivados por hacer el bien para la sociedad. Otra alternativa relevante elegida se refiere a satisfacer la curiosidad por conocer y resolver los misterios del universo. 


\section{Tabla I}

Respuestas obtenidas en la cuestión 60111.

60111. La mayoría de los científicos están motivados para esforzarse mucho en su trabajo. La razón principal de su motivación personal para hacer ciencia es:

a) Ganar reconocimiento, ya que de lo contrario su trabajo no se aceptaría.

b) Ganar dinero, porque la sociedad presiona a los científicos a esforzarse por recompensas económicas.

c) Adquirir un poco de fama, dinero y poder, porque los científicos son como todos los demás.

d) Satisfacer su curiosidad sobre el mundo natural, porque les gusta aprender más y resolver los misterios del universo físico y biológico.

e) Resolver curiosos problemas para conocimiento personal y descubrir nuevas ideas o inventar cosas para beneficio de la sociedad (p.e., curas médicas, soluciones a la contaminación, etc.). Todo esto junto representa la principal motivación de la mayoría de los científicos.

f) Desinteresadamente inventar y descubrir nuevas cosas para la ciencia y la tecnología.

g) Descubrir nuevas ideas o inventar cosas para beneficio de la sociedad (p.e., curas médicas, soluciones a la contaminación, etc.)

h) No es posible generalizar porque la motivación principal de los científicos varía de un científico a otro.
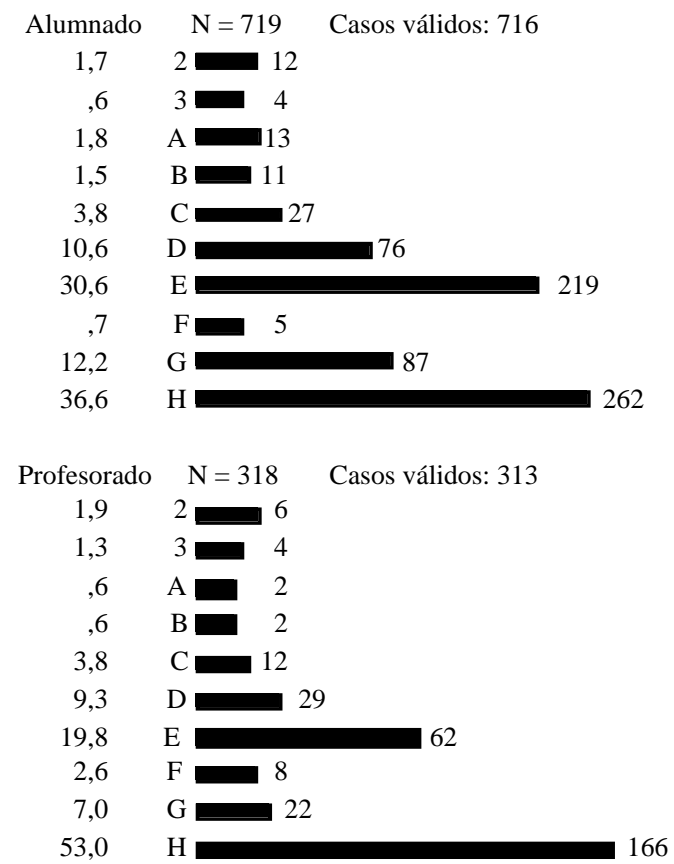

Existen diferencias estadísticamente significativas de género $(p<, 01)$, en el grupo de grado medio de exposición a la ciencia, y próximas a la significación, en el caso del grupo de bajo grado de exposición a la ciencia: las mujeres son más partidarias que los hombres de las opciones en las que los científicos actúan en beneficio de la sociedad, mientras que los hombres son más relativistas o partidarios de la curiosidad, la fama y el dinero como motivación de los científicos.

\section{Motivación de los científicos según el profesorado (60111)}

La actitud del profesorado respecto a la motivación de los científicos para hacer ciencia es mucho más clara y contundente que la del alumnado. Un gran mayoría (más de la mitad) sostiene la ambivalente actitud de considerar esta motivación como un asunto muy personal, que varía de un científico a otro, y por ello no es posible generalizar. Una cantidad relevante (un quinto) opina que la principal motivación de los científicos es descubrir e inventar para conocer y en beneficio de la sociedad. En conjunto, el perfil de actitud del profesorado es similar al del alumnado, pero la gran proporción decantada por la opción mayoritaria hace que las tasas dirigidas a otras actitudes sean considerablemente inferiores (Tabla I).

Aparecen diferencias estadísticamente significativas según la licenciatura $(\mathrm{p}<, 01)$ en el grupo alto de exposición a la ciencia, que indica que pueden existir diferencias entre científicos, según el tipo de estudios superiores realizados.

Mentalidad abierta, imparcialidad y objetividad según el alumnado (60211)

El estereotipo de la mentalidad abierta, imparcialidad y objetividad de los grandes científicos, como condiciones necesarias para su trabajo, es juzgado insuficiente por el alumnado. Una mayoría (más de un tercio) cree que, además, necesitan también tener imaginación, inteligencia y honradez. Sin embargo, las opciones que no creen necesarias estas cualidades personales consiguen un apoyo similar e, incluso, globalmente superior a la alternativa contraria. Una parte importante (un cuarto) adopta una actitud relativista: depende, unos científicos son así, pero otros están en el estereotipo contrario. Mientras otros (menos de un quinto) creen que pueden estar tan absortos en su trabajo que pueden llegar a estar en el estereotipo opuesto. Globalmente, la opinión aparece muy dividida y se puede considerar que domina la actitud contraria a que estos rasgos estereotípicos sean necesarios para hacer la mejor ciencia (Tabla II).

\section{Mentalidad abierta, imparcialidad y objetividad se- gún el profesorado (60211)}

Entre el profesorado, una mayoría más amplia (más de la mitad) cree que estos valores son necesarios pero insuficientes, necesitando también tener imaginación, inteligencia y honradez. Las opciones que no creen necesarias estas cualidades personales consiguen un apoyo inferior. Una parte importante (un quinto) adopta una actitud relativista: depende, unos científicos son así, pero otros están en el estereotipo contrario. Globalmente, el perfil de respuestas del profesorado es similar al del alumnado, 


\section{Tabla II}

Respuestas obtenidas en la cuestión 60211.

60211. El mejor científico es siempre de mentalidad abierta, imparcial y objetivo en su trabajo. Estas características personales son necesarias para hacer la mejor ciencia.

a) Los mejores científicos tienen estas características, ya que de otra forma la ciencia sufriría.

b) Los mejores científicos tienen estas características porque cuantas más características de éstas se tienen, mejor ciencia se hace.

c) Estas características no son suficientes. Los mejores científicos necesitan también otros rasgos personales, tales como imaginación, inteligencia y honradez.

No necesariamente los mejores científicos debe tener estas características:

d) Porque los mejores científicos a veces llegan a estar tan profundamente metidos, interesados o preparados en su campo, que pueden ser cerrados, parciales, subjetivos y no siempre lógicos en su trabajo.

e) Porque eso depende del carácter de cada científico. Algunos son abiertos, objetivos, etc. en su trabajo; mientras que otros pueden ser en su trabajo cerrados, subjetivos, etc.

f) Los mejores científicos tienen estas características personales en mayor medida que cualquier otro científico medio. Estas características no son necesarias para hacer ciencia.

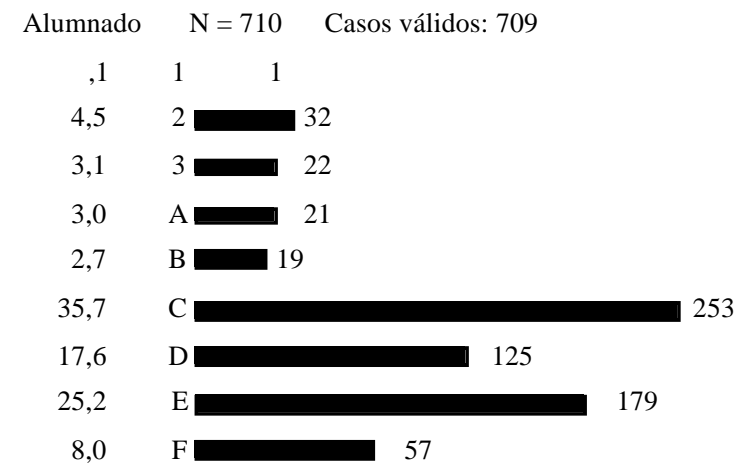

Profesorado $\mathrm{N}=336 \quad$ Casos válidos: 331

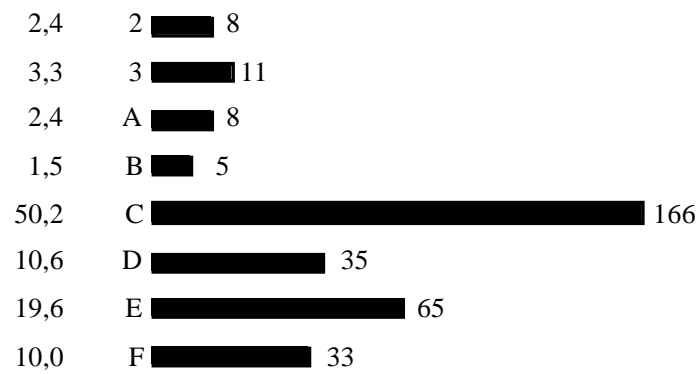

pero dada la gran mayoría alcanzada por la opción más elegida, el resultado para la actitud global es diferente: el profesorado considera que los rasgos estereotípicos propuestos son necesarios para hacer la mejor ciencia, pero que son insuficientes, mientras la mayoría del alumnado se decantaba por la actitud contraria (Tabla III).

Aparecen diferencias estadísticamente significativas según la licenciatura $(\mathrm{p}<, 01)$ en el grupo bajo de exposición a la ciencia, que indican diferencias según el tipo de estudios superiores realizados.

\section{Honradez según el alumnado (60226)}

La honradez es una pieza clave del sistema científico, pues, de otra manera, el fraude y los plagios arruinarían el progreso del conocimiento. La actitud dominante (más de la mitad) en el alumnado, respecto a la honradez de los científicos en su trabajo, es relativizar su importancia: no se puede generalizar porque los científicos son personas como los demás, de modo que unos serán honrados y otros no. El resto de las opiniones alcanza

Tabla III

Respuestas obtenidas en la cuestión 60226.

60226. Los científicos son honrados en su trabajo de investigación (p.e., cuando escriben un informe de investigación).

a) Todos, incluyendo los científicos, intentan ser honrados.

b) Los científicos son honrados en su trabajo porque sus resultados deben ser exactos y precisos.

c) Los científicos son honrados en su trabajo porque su trabajo afecta al bienestar de la sociedad.

d) Los científicos son honrados en su trabajo porque todos los resultados son comprobados por otros científicos; por tanto, necesitan ser honrados.

e) Habitualmente los científicos son honrados en su trabajo. Pero a veces falsean sus resultados por necesitar más dinero para investigar, por cumplir plazos de tiempo, por agradar al gobierno o a su jefe, o desear reconocimiento.

f) Los científicos no son honrados en su trabajo porque a veces falsean sus resultados por necesitar más dinero para investigar, por cumplir plazos de tiempo, por agradar al gobierno o a su jefe, o desear reconocimiento.

g) Algunos científicos son honrados y algunos no lo son. No se puede generalizar, porque los científicos son personas como los demás.

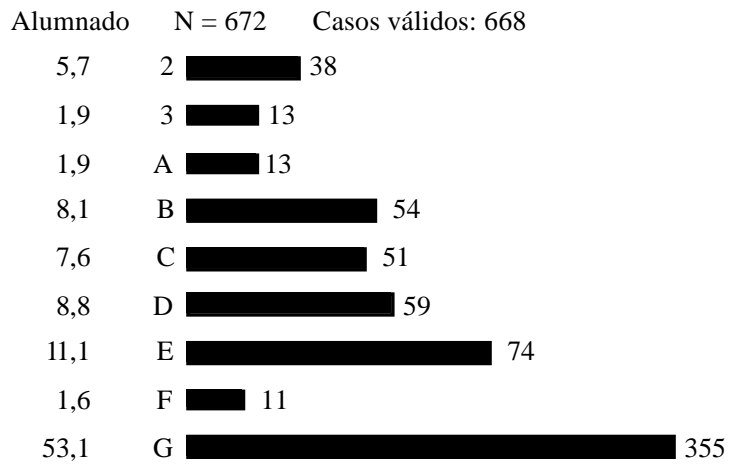


niveles bajos y similares entre sí. Quizá la actitud en esta cuestión sea una de las que ejemplifique mejor el analfabetismo científico del alumnado respecto a la ciencia y el funcionamiento de la comunidad científica como sistema de control y comprobación de los trabajos de los científicos y reclama la necesidad de una educación basada en los postulados CTS para una mejor alfabetización sobre la naturaleza de la ciencia (Tabla III).

Tabla IV

Respuestas obtenidas en la cuestión 60411.

60411. Los científicos no tienen prácticamente vida familiar o social porque necesitan estar profundamente metidos en su trabajo.

a) Los científicos necesitan estar profundamente metidos en su trabajo para tener éxito. Esta implicación profunda impide la vida social y familiar.

b) Depende de la persona. Algunos científicos están tan metidos en su trabajo, que su vida social y familiar sufren. Sin embargo, muchos científicos tienen tiempo para las cuestiones sociales y familiares.

c) En el trabajo, los científicos se toman las cosas de manera diferente que otras personas, pero esto no quiere decir que no tengan prácticamente vida familiar ni social.

La vida familiar y social de los científicos son normales:

d) De lo contrario su trabajo sufriría. La vida social es valiosa para un científico.

e) Porque muy pocos científicos están tan encerrados en su trabajo que ignoren todo lo demás.
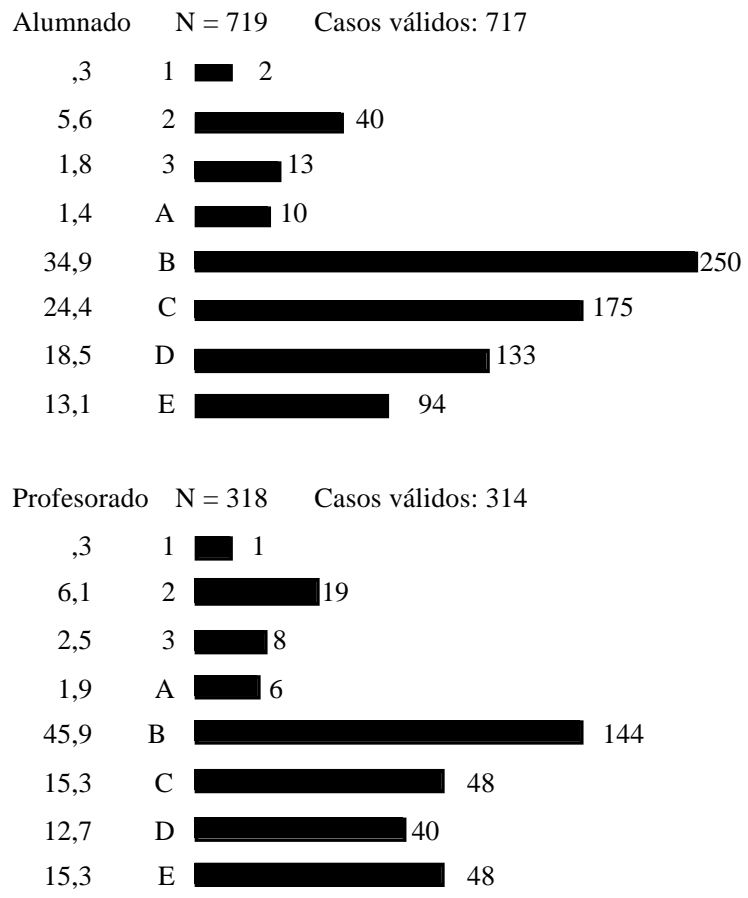

Existen diferencias estadísticamente significativas de género y edad $(\mathrm{p}<, 01)$ en el grupo de baja exposición a la ciencia. Las mujeres sostienen con mayor intensidad de la imposibilidad de generalizar sobre la honradez (la opción mayoritaria en ambos), mientras los hombres tienden a ser más partidarios de reconocer la honradez como virtud de los científicos.

Vida social y familiar de los científicos según el alumnado (60411)

El mito del científico en su torre de marfil, aislado del mundo y reconcentrado en su trabajo, es propuesto bajo la afirmación de que los científicos no tienen vida familiar y social porque necesitan estar profundamente metidos en su trabajo. Como en otras ocasiones, la actitud mayoritaria del alumnado (más de un tercio) es intermedia y relativiza la validez de esta afirmación haciéndola depender del tipo de persona: unos científicos tienen una vida familiar y social normales, mientras otros están tan metidos en su trabajo que reducen su vida social. Casi un tercio del alumnado, también, sostiene que la vida social y familiar de los científicos es normal, porque es valiosa y porque no pueden ignorarla, y un cuarto más niegan que no tengan vida social. La opción que apoya el mito de la torre de marfil recibe, pues, un apoyo insignificante. En suma, la actitud dominante del alumnado expresa que los científicos tienen una vida social y familiar normales, y sólo en algunos casos personales la dedicación al trabajo hace sufrir la vida familiar y social de los científicos (Tabla IV).

Aparecen diferencias sólo próximas al nivel de significación estadística por género y edad $(p<, 05)$ en el grupo de grado bajo de exposición a la ciencia.

Vida social y familiar de los científicos según el profesorado (60411)

También la actitud mayoritaria del profesorado (casi la mitad) es intermedia pero más intensa que entre el alumnado, haciéndola depender del tipo de persona: unos científicos tienen una vida familiar y social normales, mientras otros están tan metidos en su trabajo que reducen su vida social. Las opciones que sostienen que la vida social y familiar de los científicos son normales reúnen más de la mitad de las frecuencias, lo que significa que, globalmente, estas opciones resultan mayoritarias. La opción que apoya el mito de la torre de marfil recibe un apoyo insignificante. La actitud predominante entre el profesorado es favorable a la vida social y familiar normales de los científicos, y sólo en algunos casos personales la dedicación al trabajo repercute sobre la vida familiar y social de los científicos. Esta última posición es la mayoritaria y es más apoyada entre el profesorado que entre el alumnado (Tabla IV).

Aparecen diferencias estadísticamente significativas según el nivel educativo y el género $(\mathrm{p}<, 01)$ en el grupo de bajo grado de exposición a la ciencia (Tabla V y VI). 
Tabla V

Resultados de las respuestas de los estudiantes a la cuestión 60411 por grupos de género, controlando la variable grado de exposición a la ciencia. (Algunos datos de género omitidos ocasionan alguna pequeña diferencia con la muestra total.)

\begin{tabular}{|c|c|c|c|c|c|c|}
\hline \multicolumn{2}{|l|}{60411} & \multicolumn{5}{|c|}{ GRADO DE EXPOSICIÓN A LA CIENCIA } \\
\hline \multirow[b]{2}{*}{ Opciones } & \multicolumn{2}{|c|}{ BAJA } & \multicolumn{2}{|c|}{ MEDIA } & \multicolumn{2}{|c|}{ ALTA } \\
\hline & Hombres & Mujeres & Hombres & Mujeres & Hombres & Mujeres \\
\hline 1 & 2 & & & & & \\
\hline$\%$ col. & $1,1 \%$ & & & & & \\
\hline 2 & 14 & 15 & 4 & 4 & & \\
\hline$\%$ col. & $7,7 \%$ & $5,0 \%$ & $5,6 \%$ & $3,8 \%$ & & \\
\hline 3 & 5 & 4 & 1 & 2 & & 1 \\
\hline$\%$ col. & $2,7 \%$ & $1,3 \%$ & $1,4 \%$ & $1,9 \%$ & & $5,3 \%$ \\
\hline A & 5 & 4 & 1 & & & \\
\hline$\%$ col. & $2,7 \%$ & $1,3 \%$ & $1,4 \%$ & & & \\
\hline B & 47 & 103 & 28 & 46 & 12 & 10 \\
\hline$\%$ col. & $25,8 \%$ & $34,6 \%$ & $38,9 \%$ & $43,8 \%$ & $50,0 \%$ & $52,6 \%$ \\
\hline $\mathrm{C}$ & 42 & 89 & 13 & 21 & 3 & 3 \\
\hline$\%$ col. & $23,1 \%$ & $29,9 \%$ & $18,1 \%$ & $20,0 \%$ & $12,5 \%$ & $15,8 \%$ \\
\hline $\mathrm{D}$ & 37 & 50 & 10 & 22 & 7 & 4 \\
\hline$\%$ col. & $20,3 \%$ & $16,8 \%$ & $13,9 \%$ & $21,0 \%$ & $29,2 \%$ & $21,1 \%$ \\
\hline $\mathrm{E}$ & 30 & 33 & 15 & 10 & 2 & 1 \\
\hline$\%$ col. & $16,5 \%$ & $11,1 \%$ & $20,8 \%$ & $9,5 \%$ & $8,3 \%$ & $5,3 \%$ \\
\hline Total & 182 & 298 & 72 & 105 & 24 & 19 \\
\hline
\end{tabular}

TablaVI

Resultados de las respuestas del profesorado a la cuestión 60411 por nivel educativo, controlando la variable de grado de exposición a la ciencia.

\begin{tabular}{|c|c|c|c|c|c|c|c|c|c|}
\hline \multirow{3}{*}{$\begin{array}{l}\mathbf{6 0 4 1 1} \\
\text { Opciones }\end{array}$} & \multicolumn{3}{|c|}{ PRIMARIA } & \multicolumn{3}{|c|}{ SECUNDARIA } & \multicolumn{3}{|c|}{ UNIVERSIDAD } \\
\hline & \multicolumn{3}{|c|}{ Exposición a la ciencia } & \multicolumn{3}{|c|}{ Exposición a la ciencia } & \multicolumn{3}{|c|}{ Exposición a la ciencia } \\
\hline & BAJA & MEDIA & ALTA & BAJA & MEDIA & ALTA & BAJA & MEDIA & ALTA \\
\hline $\begin{array}{l}1 \\
\% \text { col. }\end{array}$ & $\begin{array}{c}1 \\
.7 \%\end{array}$ & & & & & & & & \\
\hline 2 & 12 & 1 & & 3 & & 2 & & & 1 \\
\hline$\%$ col. & $8,8 \%$ & $16,7 \%$ & & $4,8 \%$ & & $3,5 \%$ & & & $5,6 \%$ \\
\hline 3 & 3 & 1 & 1 & 3 & & & & & \\
\hline$\%$ col. & $2,2 \%$ & $16,7 \%$ & $20,0 \%$ & $4,8 \%$ & & & & & \\
\hline A & 3 & & & & 2 & 1 & & & \\
\hline$\%$ col. & $2,2 \%$ & & & & $10,5 \%$ & $1,8 \%$ & & & \\
\hline B & 61 & 2 & 3 & 29 & 13 & 26 & & 3 & 7 \\
\hline$\%$ col. & $44,5 \%$ & $33,3 \%$ & $60,0 \%$ & $46,0 \%$ & $68,4 \%$ & $45,6 \%$ & & $50,0 \%$ & $38,9 \%$ \\
\hline $\mathrm{C}$ & 28 & & 1 & 4 & 1 & 9 & 1 & 1 & 3 \\
\hline$\%$ col. & $20,4 \%$ & & $20,0 \%$ & $6,3 \%$ & $5,3 \%$ & $15,8 \%$ & $33,3 \%$ & $16,7 \%$ & $16,7 \%$ \\
\hline $\mathrm{D}$ & 15 & 2 & & 10 & 1 & 7 & 2 & 1 & 2 \\
\hline$\%$ col. & $10,9 \%$ & $33,3 \%$ & & $15,9 \%$ & $5,3 \%$ & $12,3 \%$ & $66,7 \%$ & $16,7 \%$ & $11,1 \%$ \\
\hline $\mathrm{E}$ & 14 & & & 14 & 2 & 12 & & 1 & 5 \\
\hline$\%$ col. & $10,2 \%$ & & & $22,2 \%$ & $10,5 \%$ & $21,1 \%$ & & $16,7 \%$ & $27,8 \%$ \\
\hline Total & 137 & 6 & 5 & 63 & 19 & 57 & 3 & 6 & 18 \\
\hline
\end{tabular}




\section{Tabla VII}

Respuestas obtenidas en la cuestión 60421.

60421. Los mejores científicos tienen la paciencia y determinación necesarias para superar la frustración y el aburrimiento (p.e., haciendo el mismo experimento muchas veces hasta obtener resultados fiables).

a) Sí, porque la frustración y el aburrimiento desafían al mejor científico para luchar y esforzarse aún más.

b) Sí, porque la paciencia y la determinación son parte del trabajo. Sin ellas, los científicos no obtendrían de ninguna manera resultados correctos.

c) No, porque ni siquiera algunos de los mejores científicos pueden afrontar la frustración. Los científicos tienen grados variables de paciencia, como cualquier otra persona.

d) No, porque los mejores científicos NO son suficientemente inteligentes para evitar la frustración y el aburrimiento. Frustración y aburrimiento hacen más difícil para cualquiera llegar al éxito.

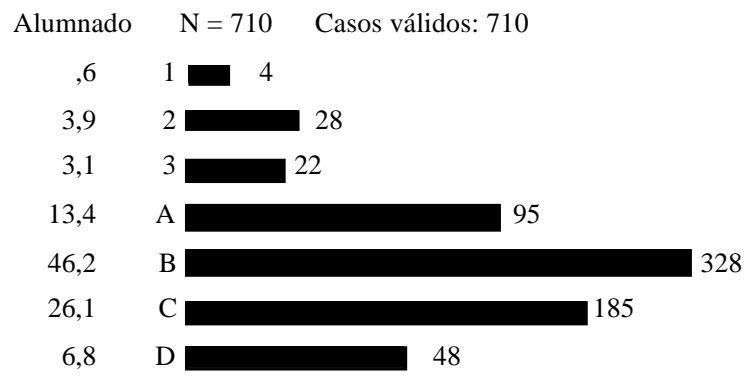

Profesorado $\mathrm{N}=336 \quad$ Casos válidos: 332

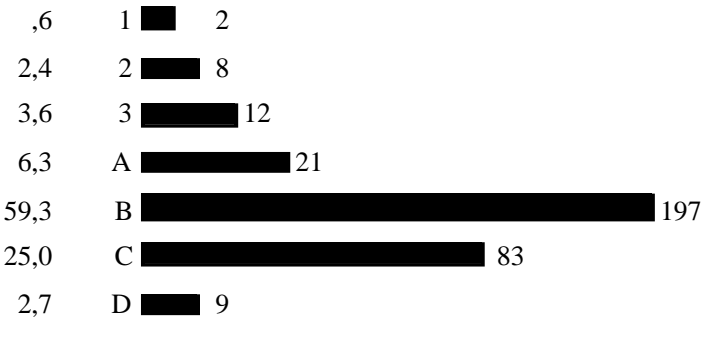

\section{Paciencia y determinación de los científicos según el alumnado (60421)}

El trabajo científico requiere paciencia y constancia para superar los momentos de frustración, -cuando no se alcanzan buenos resultados-y de aburrimiento, ante el tedio de repetir comprobaciones numerosas veces. La actitud mayoritaria del alumnado (menos de la mitad) cree que sin paciencia y determinación el trabajo científico no podría alcanzar resultados correctos. La segunda actitud mayoritaria (un cuarto) es contraria, pues considera que los científicos tienen la misma capacidad de paciencia y determinación que el resto de las personas y ni siquiera los mejores científicos son capaces de supe- rar la frustración. Globalmente, las actitudes que consideran la paciencia y la constancia como capacidades de los científicos superan ampliamente la mitad, mientras sólo un tercio sostienen la actitud contraria (Tabla VII).

Existen diferencias estadísticamente significativas de género $(\mathrm{p}<, 01)$ en el grupo de baja exposición a la ciencia; las mujeres de bajo grado de exposición a la ciencia sostienen con mayor intensidad que paciencia y determinación no son cualidades de los científicos, mientras los hombres tienden a ser más partidarios de reconocer la paciencia y la determinación como virtudes de los científicos.

\section{Paciencia y determinación de los científicos según el profesorado (60421)}

La actitud mayoritaria del profesorado (más de la mitad) sostiene que, sin paciencia y determinación, el trabajo científico no podría alcanzar resultados correctos. La segunda actitud mayoritaria (un cuarto) es contraria, pues considera que los científicos tienen la misma capacidad de paciencia y determinación que el resto de las personas y ni siquiera los mejores científicos son capaces de superar la frustración. Globalmente, las actitudes que consideran la paciencia y la constancia como capacidades de los científicos son más dominantes (dos tercios) todavía entre el profesorado que entre el alumnado, mientras sólo poco más de un cuarto sostiene la actitud contraria (Tabla VII). Aparecen diferencias estadísticamente significativas según licenciatura $(\mathrm{p}<, 05)$ en el grupo de grado bajo de exposición a la ciencia.

\section{DISCUSIÓN}

La aportación del estudio en el nivel descriptivo está directamente relacionada con los contenidos actitudinales de la enseñanza de las ciencias y, en este caso, referidos a las características del trabajo de los científicos. A pesar de que tanto el movimiento CTS como la propuesta de alfabetización científica son las dos líneas de progreso más innovadoras en la enseñanza de la ciencia actual que enfatizan la enseñanza de las actitudes relacionadas con la ciencia, éstas son manifiestamente desatendidas en la escuela, en favor de la más tradicional enseñanza de conceptos y principios. Debe enfatizarse que el objetivo del estudio global de donde se han extraído los resultados expuestos es diagnosticar las actitudes de todos los jóvenes sobre estas cuestiones CTS que no habían sido explícitamente atendidas en los currículos de ciencias.

El análisis de las actitudes en temas relacionados con la ciencia y la tecnología suele emplear casi siempre muestras de alumnado y profesorado de ciencias, porque se consideran cuestiones para tratarse en la educación a través de las asignaturas de ciencias, que afectan al alumnado (de ciencias) y al profesorado (de ciencias). Sin embargo, el interés por la ciencia y la tecnología, 
debido a su penetrante influencia en la sociedad, no debería estar reducido al alumnado que se educa en un aula de ciencias, sino que interesa a todos los ciudadanos; por tanto, no son sólo los estudiantes de ciencias quienes deben ser encuestados y educados, sino todos los estudiantes, científicos y no científicos, que serán ciudadanos mañana y tendrán una responsabilidad cívica en la toma de decisiones sociotécnicas, para las que necesitan una mínima alfabetización científica. Desde esta perspectiva de alfabetización debe promoverse una enseñanza de la ciencia para todos, para los estudiantes de ciencias y para los estudiantes de letras, con el objetivo de que todos aprendan sobre ciencia más que aprender mucha ciencia. Los estudios basados en muestras sólo de formación científica son sesgadas, y por ello tienen una validez limitada, pues la homogeneización muestral tiene el efecto de sesgar los resultados en la dirección de la muestra, olvidando la parte del alumnado que también debe tener acceso a saber de ciencia. Por ello, en este estudio, las muestras de profesorado y alumnado contienen personas con formación científica y no científica, por lo que las opiniones obtenidas pueden resultar realmente representativas de la población general.

La cuestión del profesorado y la educación de las actitudes sobre ciencia debe considerar la gran diversidad de profesores que participan en la enseñanza de la ciencia en el sistema educativo, unos con formación específicamente científica, en el bachillerato y la universidad, pero otros en la educación primaria y los primeros años de secundaria carecen de una formación específicamente científica. Sin disminuir el papel central del profesorado de ciencias de secundaria y su formación, las actitudes relacionadas con la ciencia y la tecnología son enseñadas y trasmitidas, implícita o explícitamente, por todo el profesorado, científico y no científico, ya que no sólo el profesorado de las materias de ciencias puede contribuir a crear actitudes y opiniones adecuadas en relación con la ciencia y la tecnología. Respecto al profesorado, no obstante, el principal problema de unos y otros es la falta de una formación inicial específica en temas de CTS o de historia, sociología y filosofía de la ciencia. En el modelo actual de la formación inicial de los maestros, por ejemplo, éstos no sólo no reciben formación epistemológica sino que tampoco reciben una formación especializada en ciencias; si convenimos que su papel con el alumnado más joven es también crucial, no tanto para enseñar conceptos de ciencia como para una comprensión de la ciencia, la situación parece deplorable y claramente necesitada de mejora. Por otro lado, la formación epistemológica del profesorado de ciencias no es uniforme, siendo en la mayoría de los casos una formación implícita, a través de las prácticas de aprendizaje realizadas en sus respectivas carreras, y no una educación reglada, intencional, planificada y directa, de modo que su formación inicial en estas cuestiones resulta sesgada por las convicciones de sus profesores y manifiestamente mejorable. La ausencia de diferencias importantes entre alumnado y profesorado y entre los grupos de exposición a la ciencia del profesorado encontradas en este estudio, de alguna manera, constituyen indicadores importantes de las profundas deficiencias de la formación inicial del profesorado en estas cuestiones.
Las actitudes del alumnado respecto a la motivación de los científicos para investigar son favorables a considerar una motivación epistemológica (para conocer) y altruista (beneficiar a la sociedad) en el quehacer de los científicos y también consideran que la determinación es parte del trabajo de los científicos, sin la cual no podrían obtener resultados correctos. En el caso de la mentalidad abierta, imparcialidad y objetividad, el alumnado, globalmente, no cree que estas cualidades sean esenciales para el científico y, en el caso de la honradez, la actitud general es ambivalente y escéptica. Las relaciones sociales de los científicos se consideran normales, similares a las de cualquier otra persona y, por tanto, alejadas del mito de la torre de marfil.

En conjunto, el profesorado sostiene actitudes similares al alumnado, si acaso con una mayor intensidad o definición; es decir, el perfil general de las distribuciones de respuestas sobre las opciones son similares entre profesorado y alumnado, pero las actitudes dominantes del profesorado son sostenidas con mayor apoyo que las del alumnado. Éste es el caso de las actitudes referidas a la motivación de los científicos, la honradez y la paciencia y la determinación. La única excepción es el caso de la mentalidad abierta, la imparcialidad y la objetividad, en que los estudiantes no las consideran mayoritariamente como características de los científicos, mientras que sílo hacen los profesores.

En principio, estos resultados parecen de sentido común y, como todas las concepciones de sentido común, suscitarían un acuerdo muy general entre los no-especialistas. Sin embargo, desde una perspectiva especializada basada en el marco teórico de la sociología de la ciencia, debe constatarse la ingenuidad de algunas respuestas mayoritarias y su contrapartida de fracaso para descubrir aspectos básicos de los valores de la comunidad científica. Por ejemplo, la búsqueda del reconocimiento de los científicos (60111, opción A: 1,8\% alumnado, 0,6\% profesorado) y, en otro nivel, el desinterés (opción F: $0,7 \%, 2,6 \%$ ) apenas son identificadas; la honradez (60226) se justifica con un heurístico (todos somos iguales), pero no se capta que la necesidad de financiación, satisfacer al jefe o desear el reconocimiento personal (opción E: $11,1 \%$ ), a veces, pueden hacer flaquear la honradez; por otro lado, el mismo heurístico (todos somos iguales) se aplica menos para explicar la paciencia y determinación de los científicos cuando investigan (60421, opción C: $26 \%, 25 \%)$.

Los estudiantes canadienses de secundaria del estudio original de Aikenhead y Ryan (1989) ofrecen también una referencia relativa para comparar las actitudes obtenidas aquí, teniendo presente que ambas muestras no son equivalentes y, por ello, no se pueden hacer definitivas las conclusiones sobre las diferencias. No obstante, la mayor heterogeneidad de nuestra muestra, en edad y estudios, le confiere una mayor representatividad respecto a la población general. Los resultados canadienses están explicitados para francófonos y anglófonos, cuyas diferencias entre sí superan a veces las diferencias con los españoles, por lo que es difícil decidir diferencias significativas. En la vida social y paciencia de los cien- 
tíficos, los canadienses y nuestra muestra prácticamente tienen el mismo perfil de distribución de respuestas. Análogamente sucede para la objetividad, donde se pueden observar pequeñas diferencias: nuestra muestra elige un poco más que los canadienses respecto a que los científicos pueden no ser tan objetivos, etc. En lo que se refiere a la motivación de los científicos, los canadienses y españoles tienen el mismo perfil de distribución de respuestas, con una diferencia cuantitativa en las dos opciones mayoritarias: los canadienses apoyan más la opción de conocimiento personal y beneficio de la sociedad y menos que la motivación varía de científico a científico. Finalmente, las diferencias son más notables en las actitudes sobre la honradez de los científicos, más apoyada por los canadienses que por los españoles, que tienden a considerarlos más como cualesquiera otras personas, algunos honrados y otros menos. Se podría decir que, en conjunto, los canadienses tienden a apoyar más que nuestra muestra algunos de los rasgos del estereotipo de los científicos, especialmente la honradez, la objetividad y la motivación por el conocimiento. Algunos resultados habían sido ya informados por estos autores en el estudio empírico previo que fue parte de la génesis del VOSTS, aunque con cuestiones no estrictamente equivalentes a las usadas en estas versiones definitivas (Aikenhead, 1987; Ryan, 1987) y replicadas también en otros estudios españoles. Los estudiantes de bachillerato (296) y universidad (172) de Borreguero y Rivas (1995) opinaron mayoritariamente que los científicos tienen menos que otras personas en su trabajo las características de honestidad y tolerancia. En otra muestra de secundaria y universidad (945) se opina que la principal motivación de los científicos es satisfacer la curiosidad, que el trabajo de los científicos no requiere más objetividad e imparcialidad que otros trabajos y que honradez y objetividad son resultado del control de la comunidad científica (Vázquez y Manassero, 1998b).

Desde la perspectiva de la educación en ciencias, este estudio es un diagnóstico que puede ser útil al profesorado como una evaluación inicial de las actitudes y opiniones previas del alumnado sobre cuestiones relacionadas con la comprensión de la ciencia y la tecnología en la sociedad (CTS). El profesorado puede utilizarlo como guía para conocer las ideas previas del alumnado o intentar evaluar él mismo las ideas de sus propios estudiantes utilizando la cuestiones aplicadas aquí. En otro nivel, las cuestiones también pueden servir como instrumento de desarrollo curricular en la clase de los temas de sociología interna de la ciencia, por ejemplo, como guías para actividades de discusión sobre el significado y las implicaciones de todas las alternativas de respuesta que se proponen para cada cuestión (Vázquez, 1999).

Finalmente, este diagnóstico de las actitudes de estudiantes y profesorado sugiere una reflexión sobre las consecuencias para la enseñanza de la ciencia. Inspirada por la epistemología positivista, la enseñanza tradicional de la ciencia tiende a presentar hechos, conceptos y principios de la ciencia como verdades objetivas, incontrovertibles y la metodología científica como un algorit- mo repetible cíclicamente, separando artificialmente teoría y práctica, y excluyendo deliberadamente los valores contextuales al borrar sistemáticamente la historia de la ciencia en los libros de texto. Este enfoque produce currículos científicos descontextualizados, dominados por la lógica de la ciencia y por los contenidos conceptuales, los cuales, por un lado, ignoran las experiencias y los conocimientos previos de los estudiantes en los que se produce una formación abstracta, alejada de la vida diaria de los estudiantes y, por otro lado, excluyen esos contenidos de los progresos sobre la ciencia obtenidos de los estudios de historia, epistemología y sociología de la ciencia.

Para evitar estos defectos, el tema educativo de fondo de este trabajo plantea una visión comprensiva de la enseñanza de la ciencia y la tecnología en un contexto social, es decir, interaccionando con la sociedad que permita a los estudiantes, no tanto saber mucha ciencia, como entender la ciencia. El movimiento ciencia-tecnologíasociedad para la innovación en la enseñanza de la ciencia propone un enfoque contextualizado, basado en resaltar las relaciones mutuas entre ciencia, tecnología y sociedad y promover una visión global de los problemas como complejos procesos de toma de decisiones, y adaptándose a la personalidad y experiencias sociales de los estudiantes. La reforma contextualizada de la enseñanza de la ciencia (CTS) no es fácil, pues la enseñanza tradicional de la ciencia, a pesar de sus evidentes fracasos, se halla bien implantada en la percepción dominante del público y del profesorado por su simplicidad (Koul y Dana, 1997). Los cambios curriculares hacia la reforma son complejos, pues no sólo requieren la puesta a punto de materiales nuevos, sino la transformación global del sistema educativo (diseño curricular, evaluación y exámenes, formación del profesorado, etc.). Por eso, cuando mucho debates de la didáctica de la ciencia se centran en la metodología de los aprendizajes y las teorías del aprendizaje, debe reiterarse que algunas cuestiones básicas del diseño curricular de ciencias están todavía sin abordarse explícitamente, como, por ejemplo, todo lo que afecta a la definición de objetivos y contenidos curriculares relacionados con la cuestión CTS. En el currículo español, son explícitas las propuestas CTS en el bachillerato (aunque es preocupante la evidente actitud reaccionaria de algunos libros de texto), pero son muy implícitas y necesitadas de concreción en los otros niveles educativos obligatorios, que, para una gran parte de la población, son su único contacto con la ciencia y la única ocasión de lograr una mínima alfabetización científica.

Debido a la abrumadora influencia de la ciencia en las vidas de las personas de todo el mundo, es necesario ayudar a la gente a entenderla, desde una perspectiva tanto de crítica como de aprecio, pero no desde un marco de aislamiento de la cultura científica en sí misma, sino abriéndose a otras disciplinas (Carson, 1997), como también se sugiere aquí en relación con la educación CTS y la línea de alfabetización científica o ciencia para todos. En este punto debe reiterarse la necesidad de incluir contenidos históricos, filosóficos, sociológicos, psicológicos y culturales sobre la ciencia (Campanario, 
1999) para hacer efectiva la comprensión de ésta por los estudiantes de todos los niveles educativos, y para conseguir una preparación real y una ayuda básica para la toma de decisiones en su vida diaria y en el ejercicio responsable de la ciudadanía.

Los resultados mostrados son una primera aproximación a estas cuestiones todavía poco comunes en la literatura, incluso en didáctica de las ciencias. El objetivo general de diagnóstico de actitudes no ha permitido entrar en los muchos detalles de interés que pueden plantearse desde una perspectiva de investigación didáctica, sino más bien incidir en las deficiencias que ponen de manifiesto

\section{REFERENCIAS BIBLIOGRÁFICAS}

AIKENHEAD, G.S. (1987). High School Graduates' Beliefs About Science-Technology-Society. III. Characteristics and Limitations of Scientific Knowledge. Science Education, 71(4), pp. 459-487.

AIKENHEAD, G.S. (1988) An analysis of four ways of assessing student beliefs about STS topics. Journal of Research in Science Teaching, 25, pp. 607-627.

AIKENHEAD, G.S. y RYAN, A.G. (1989). The development of a multiple choice instrument for monitoring views on Science-Technology-Society topics. Final Report of SSHRCC Grant: Autor.

AIKENHEAD, G.S. y RYAN, A.G. (1992). The development of a new instrument: «Views on Science-Technology-Society» (VOSTS). Science Education, 76(5), pp. 477-492.

AIKENHEAD, G.S., RYAN, A.G. y FLEMING, R.G. (1989). Views on Science-Technology-Society Form CDN.MC.5. Department of Curriculum Studies College of Education, Autor.

BOYLAN, C.R., HILL, D.M., WALLACE, A.R. y WHEELER, A.E. (1992). Beyond stereotypes. Science Education, 76, pp. 465-476.

BORREGUERO, P. y RIVAS, F. (1995). Una aproximación empírica a través de las relaciones ciencia-tecnología-sociedad (CTS), en estudiantes de secundaria y universitarios valencianos. Enseñanza de las Ciencias, 13, pp. 363-370.

CAMPANARIO, J.M. (1999). La ciencia que no enseñamos. Enseñanza de las Ciencias, 17, pp. 397-410.

CARSON, R.E. (1997). Why science education alone is not enough? Interchange, 28, pp. 109-120.

DE SOLLA-PRICE, D.J. (1973). Hacia una ciencia de la ciencia. Barcelona: Ariel.

GIL, D. (1993). Contribución de la historia y de la filosofía de las ciencias al desarrollo de un modelo de enseñanza-aprendizaje como investigación. Enseñanza de las Ciencias, 11, pp. 197212.

KAHLE, J. (1989). Images of scientists: Gender issues in science classroom. Curtin University, Key Paper, 1. Perth: Center for School Science and Mathematics. estos resultados respecto a la formación de las actitudes, tanto desde la perspectiva de la enseñanza escolar como de la formación del profesorado, para reclamar la atención sobre ellas.

\section{NOTA}

Este estudio ha sido financiado por el Centro de Investigación, Documentación y Evaluación(CIDE) del Ministerio de Educación a través de las ayudas a la investigación educativa de 1995 .

\section{AGRADECIMIENTOS}

Nuestro agradecimiento a dos revisores anónimos, cuyas sugerencias han contribuido a mejorar este artículo.
KNORR-CETINA, K. (1981). The manufacture of knowledge. Oxford: Pergamon Press.

KOUL, R. y DANA, T. (1997). Contextualized science for teaching science and technology. Interchange, 28, pp. 121144.

LAMO, E., GONZÁLEZ, J.M. y TORRES, C. (1994). La sociología del conocimiento y de la ciencia. Madrid: Alianza Editorial.

MASON, C.L., KAHLE, J.B. y GARDNER, A.L. (1991). Draw-a-scientist test: Future implications. School Science and Mathematics, 91, pp. 193-198.

MATTHEW, J.A.D. (1991). Cartoons in Science. Physics Education, 26, pp. 110-114.

MATTHEWS, B. (1996). Drawing scientists. Gender and Education, 8, pp. 231-243.

McADAM, J.E. (1990). The persistent stereotype: children's images of scientists. Physics Education, 25, pp. 102-105.

MERTON, R.K. (1977). La sociología de la ciencia. Madrid: Alianza Editorial

MITROFF, I. (1974). Norms and counter-norms in a selected group of the Apollo Moon Scientists: A case study of the ambivalence of scientists. American Sociological Review, 39, pp. 579-595.

NEWTON, D.P. y NEWTON, L.D. (1992). Young children's perceptions of science and scientists. International Journal of Science Education, 14(3), pp. 331-348.

REICHENBACH, H. (1966) La filosofía científica. México: FCE.

RYAN, A.G. (1987). High School Graduates' Beliefs About Science-Technology-Society. IV. The Characteristics of Scientists. Science Education, 71(4), pp. 489-510.

SÁNCHEZ RON, J.M. (1992). El poder de la ciencia. Historia socioeconómica de la física (siglo XX). Madrid: Alianza Editorial.

SCHIBECI, R.A. (1986). Images of science and scientists and science education. Science Education, 70, pp. 139-149. 
SCHIBECI, R.A. y SORENSON, I. (1983). Elementary school children's perceptions of scientists. School Science and Mathematics, 83, pp. 14-20.

SNOW, C.P. (1959). The two cultures and the scientific revolution. Nueva York: Cambridge University Press. [1987, Las dos culturas, Madrid, Alianza Editorial].

VÁZQUEZ, A. y MANASSERO, M.A. (1995). Actitudes relacionadas con la ciencia: unarevisión conceptual.Enseñanza de las Ciencias, 13(3), pp. 337-346.

VÁZQUEZ, A. y MANASSERO, M.A. (1996). Factores determinantes de las actitudes relacionadas con la ciencia. Revista Española de Pedagogía, 203, pp. 43-78.

VÁZQUEZ, A. y MANASSERO, M.A. (1997). Actitudes y valores relacionados con la ciencia, la tecnología y la sociedad en alumnado y profesorado. Implicaciones para la educación de las actitudes. Memoria final de investigación. Madrid, MEC-CIDE.

VÁZQUEZ, A. y MANASSERO, M.A. (1998a). Dibuja un científico: imagen de los científicos en estudiantes de secundaria. Infancia y Aprendizaje, 81, pp. 3-26.

VÁZQUEZ, A. y MANASSERO, M.A. (1998b). Actituds de l'alumnat relacionades amb la ciència, la tecnologia $i$ la societat. Palma de Mallorca: Conselleria d'Educació, Cultura i Esports.

VÁZQUEZ, A. (1999). Innovando la enseñanza de las ciencias: El movimiento ciencia-tecnología-sociedad. Revista del Col-legi Oficial de Doctors i Llicenciats de Balears, 8, pp. 25-35.

WEART, S. (1988). The physicist as mad scientists. Physics Today, June, pp. 28-37.

[Artículo recibido en diciembre de 1999 y aceptado en octubre de 2000.] 